\title{
Dieta cetogénica en epilepsia refractaria: Eficacia, evolución y complicaciones a largo plazo
}

\author{
XIMENA RAIMANN T. ${ }^{1}$, VERÓNICA MARÍN B. ${ }^{1}$, VERÓNICA BURÓN K. ${ }^{2}$, \\ MARCELO DEVILAT B ${ }^{2}$, AMANDA UGALDE F. ${ }^{3}$ \\ 1. Pediatra. Unidad de Nutrición, Hospital Luis Calvo Mackenna. Departamento Pediatría y Cirugía Infantil Oriente, \\ Facultad de Medicina, Universidad de Chile. \\ 2. Neurólogo infantil. Servicio de Psiquiatría y Neurología, Hospital Luis Calvo Mackenna. \\ 3. Nutricionista. Servicio de Alimentación, Hospital Luis Calvo Mackenna.
}

\begin{abstract}
Ketogenic Diet in Refractory Epilepsy: efficacy, evolution and long-term complications

Background: Ketogenic diet (KD) represents an alternative in treatment of refractory epilepsy (RE). Objective: To evaluate the efficacy of the diet and the frequency of complications in patients belonging to the KD Program from Luis Calvo Mackenna Children's Hospital (HLCM). Methods: Evaluation of all children enrolled in the program between 1999 and 2004, with analysis every 6 months of the diet efficacy, digestive tolerance, nutritional status, cholesterol levels and nephrolithiasis. Results: 21 children were admitted, 14 boys, age between 6 months -17 years-old. $76 \%, 71 \%$ and $67 \%$ of patients followed KD at 6 , 12 and 18 months, respectively, with KD efficacy of $67 \%$. At 12 months, $24 \%$ of patients did not present seizures. At 18 months, $85 \%$ remained close to ideal body weight ( $15 \%$ obesity) and height/age $\mathrm{Z}$ score decreased $(-0,7 \pm 0,4 ; \mathrm{p}<0,05)$. Total cholesterol significantly increased at 6 months $(64 \%$ hypercholesterolemia; decreased to $15 \%$ at 18 months). 2 patients developed nephrolithiasis. Conclusions: The study shows high efficacy of the KD for treatment of refractory epilepsy, with low rate of complications. It should be considered as a therapeutic alternative for these patients.

(Key words: ketogenic diet, refractory epilepsy, seizures, children).

Rev Chil Pediatr 2007; 78 (5): 477-481
\end{abstract}

\section{RESUMEN}

La dieta cetogénica (DK) es una opción de tratamiento en epilepsia refractaria (ER). En Chile no hay estudios publicados al respecto. Objetivo: Evaluar la eficacia de la dieta en el control de las convulsiones y la frecuencia de complicaciones en los pacientes del programa de DK, para el tratamiento de ER, del Hospital Luis Calvo Mackena (HLCM). Pacientes y Método: Evaluamos todos los niños ingresados al programa entre 1999-2004. Para efectos de este trabajo se consideró el control al ingreso y cada 6 meses, evaluándose: eficacia de la dieta, tolerancia digestiva, evolución nutricional, niveles de colesterol plasmático

Trabajo recibido el 16 de mayo de 2007, devuelto para corregir el 20 de junio de 2007, segunda versión el 9 de agosto de 2007, aceptado para publicación el 10 de septiembre de 2007.

Correspondencia a:

Dra. Ximena Raimann T.

E-mail: xraimann@mi.cl 
y litiasis renal. Resultados: Ingresaron 21 niños de 6,2 años (6 meses a 17 años), 14 de sexo masculino. A los 6,12 y 18 meses, 76\%, 71\% y 67\% de los pacientes, respectivamente, se mantenía en dieta. La eficacia del tratamiento fue $67 \%$. A los 12 meses, $24 \%$ de los pacientes estaba sin crisis. A los 18 meses $85 \%$ de los pacientes estaba eutrófico y $15 \%$ obeso. Se observó deterioro en la talla (delta zT/E $-0,7 \pm 0,4 ; \mathrm{p}<0,05$ ). El colesterol total aumentó significativamente a los 6 meses, encontrándose el $64 \%$ hipercolesterolémico; a los 18 meses este porcentaje se redujo a 15\%. Dos pacientes presentaron litiasis renal (9\%). Conclusiones: Este estudio muestra una muy buena eficacia de la dieta cetogénica para el tratamiento de la epilepsia refractaria, y una baja frecuencia de complicaciones, por lo que debería ser considerada como alternativa terapéutica en estos pacientes.

(Palabras clave: dieta cetogénica, epilepsia refractaria, convulsiones, niños).

Rev Chil Pediatr 2007; 78 (5): 477-481

\section{Introducción}

Epilepsia refractaria (ER) o intratable es aquella definida por un inadecuado control de las convulsiones a pesar de un óptimo tratamiento con medicamentos convencionales.

La dieta cetogénica (DK) es una dieta alta en grasa, adecuada en proteínas y muy baja en hidratos de carbono, que ha sido usada por más de 80 años para el tratamiento de la ER ${ }^{1}$. Originalmente esta dieta fue diseñada para imitar los cambios bioquímicos asociados con el ayuno, el cual había demostrado asociación con disminución en las convulsiones. Con la aparición de nuevos medicamentos anticonvulsivantes, la dieta cayó en desuso y la falta de experiencia llevó a la opinión que ésta no era de utilidad y era mal tolerada ${ }^{2}$. Desde principios de la década del noventa la DK nuevamente ganó popularidad como una opción de tratamiento, demostrándose en varios estudios en el mundo que, aproximadamente la mitad de los pacientes, disminuye un $50 \%$ el número de convulsiones, y cerca de un tercio del total disminuye un $90 \%$ su número de crisis ${ }^{3}$.

Se han descrito complicaciones de aparición temprana (deshidratación, hipoglicemia, vómitos, acidosis metabólica) y otras tardías, como constipación, hipercolesterolemia, litiasis renal, miocardiopatía, pancreatitis y trastorno de la coagulación $^{4-12}$.

En Chile, no hay datos publicados al respecto, por lo que el objetivo de este estudio fue evaluar prospectivamente la eficacia de la dieta en el control de las convulsiones y la frecuencia de complicaciones en los pacientes ingresados a nuestro programa de DK, para el tratamiento de una ER.

\section{Pacientes y Método}

Se evaluaron todos los niños que ingresaron entre enero de 1999 y abril de 2004, al programa de DK del Hospital Luis Calvo Mackenna (HLCM) para tratamiento de una ER y que fueron seguidos, al menos 6 meses, en los Policlínicos de Neurología y Nutrición. Se solicitó consentimiento escrito e informado de los padres o tutores. La edad de los niños fluctuaba entre 6 meses y 17 años. La mayoría de los pacientes tenía convulsiones diarias, a pesar de un adecuado tratamiento con al menos 2 anticonvulsivantes. A todos los pacientes se les hizo una evaluación nutricional, previo al ingreso y, si estaban desnutridos, debieron ser recuperados antes de iniciar la DK. Además se les solicitó perfil lipídico, para descartar hipercolesterolemia y orina completa, para evaluar la presencia de elementos sugerentes de litiasis como glóbulos rojos o cristales.

Todos los niños fueron hospitalizados para la inducción de la cetosis. La dieta cetogénica se inició con un período de ayuno de 24 a 72 horas y aporte hídrico equivalente al $75-80 \%$ de los requerimientos, con control de cetonuria cada 6 horas. Una vez lograda la cetonuria de tres cruces $(28 \mathrm{mmol} / \mathrm{L})$ se inició la dieta propiamente tal con un tercio del aporte de energía el primer día, dos tercios el segundo y el aporte energético total el tercer día. La relación calorías grasas: calorías no grasas usada fue mayo- 
ritariamente $4: 1$, pero en tres pacientes se usó 3,5:1 y, en dos, 3:1. La dieta se suplementó con multivitamínicos y calcio. Los pacientes se mantuvieron hospitalizados el tiempo necesario para evaluar tolerancia a la dieta y educar a la familia para la adecuada preparación de la alimentación en el hogar.

El seguimiento ambulatorio se hizo en los policlínicos de nutrición y neurología, mensualmente los primeros 2 meses y luego cada 3 meses. Para efectos de este trabajo se consideró el control clínico y de laboratorio realizado al ingreso y luego cada 6 meses.

Evaluamos: eficacia de la dieta, definiéndose como tal la disminución de la frecuencia de convulsiones en al menos 50\%; intolerancia digestiva (vómitos o diarrea atribuibles a la DK); evolución del estado nutricional, usando índices peso/talla y talla/edad; frecuencia de hipercolesterolemia mediante perfil lipídico (método colorimétrico) y litiasis renal (ecografía renal, en pacientes con elementos sugerentes en el examen de orina).

Mientras los pacientes estuvieron con DK no se agregaron nuevos medicamentos anticonvulsivantes.

Análisis Estadístico: se usó promedio y desviación estándar o medianas y rangos; $\mathrm{t}$ de Student para comparación de promedios y Test de Fisher para evaluar diferencias de frecuencia.

\section{Resultados}

Ingresaron 21 niños, de los cuales 15 se mantuvieron al menos 1 año en dieta.

Del total de pacientes, 14 eran de sexo masculino. La mediana de edad al inicio de la dieta fue de 6,2 años (6 meses a 17 años). Con respecto al estado nutricional al ingreso, $67 \%$ de los pacientes estaba eutrófico, $9 \%$ sobrepeso y $24 \%$ en riesgo de desnutrir. El promedio de talla al ingreso estaba dentro de lo normal (zT/E $-0,7 \pm 1,2)$. Había 3 pacientes con talla baja (menor a 2 DS). El diagnóstico neurológico fue de Epilepsia sintomática en 20 casos y criptogénica en 1 paciente. El número de convulsiones diarias, antes de iniciar la DK, fueron: incontables en 6 pacientes, 5-20 convulsiones en 9 pacientes, $2-5$ en 4 y menos de 1 convulsión al día en 2 niños. Previo al inicio de la DK cada paciente había usado un promedio de 5,8 anticonvulsivantes. Al momento de iniciarla, recibían 2,6 medicamentos.

A los 6 meses de seguimiento, 16 pacientes $(76 \%)$ se mantenían en dieta. De los 5 pacientes restantes, 1 falleció por una causa ajena a la DK, un niño no pudo continuar por diarrea persistente y los otros 3 la abandonaron, por dificultades en la elaboración y cumplimiento de la dieta. A los 12 meses, 15 pacientes (71\%) seguían en dieta y 1 suspendió por fracaso. A los 18 meses otro paciente había suspendido por estatus epilépticos sucesivos. Los 15 pacientes que completaron más de 1 año en DK, estuvieron 2,6 años (1-6,3 años) con la dieta.

La eficacia del tratamieto fue $67 \%$. A los 6 meses, 2 pacientes estaban sin crisis; en 8 niños habían disminuido en más del 90\%; en 3 habían disminuido a la mitad y 3 no habían cumplido el criterio de respuesta, pero la familia quiso continuar con la dieta, porque los niños estaban más conectados al medio y la disminución del número de convulsiones era importante para ellos. A los 12 meses, el número de pacientes sin crisis había aumentado a $5(24 \%)$ y a los 18 meses se mantenía esta respuesta (tabla 1). En el seguimiento posterior a los 18 meses de tratamiento, se suspendió la DK en forma programada en 10 pacientes, sin aumento de convulsiones en 7 . De éstos, cuatro se mantuvieron libres de crisis. En 3 niños aumentaron las convulsiones mientras se disminuía la dieta y uno de éstos decidió retomarla, completando hasta ahora más de 6 años en dieta.

En cuanto a la evolución del estado nutricional, a los 6 meses 3 pacientes se habían desnutrido $(\mathrm{P} / \mathrm{T}<-2 \mathrm{DE})$, pero a los 18 meses ya se habían recuperado, encontrándose un $85 \%$ de los pacientes eutróficos y un $15 \%$ obesos. A los 18 meses de seguimiento se observó un deterioro significativo en la talla para la edad (delta zT/E $-0,7 \pm 0,4 ; \mathrm{p}<0,05)$.

Con respecto al perfil lipídico, al ingreso $39 \%$ de los pacientes tenía CT considerado de riesgo (170-199 mg/dl) y no había hipercolesterolemia. A los 6 meses de iniciada la dieta cetogénica el CT aumentó en forma significativa $(163,4 \pm 25,4 \mathrm{mg} / \mathrm{dl}$ vs $221 \pm 47 \mathrm{mg} / \mathrm{dl}$, 
Tabla 1. Eficacia de la dieta cetogénica (DK)

\begin{tabular}{|c|c|c|c|c|c|c|}
\hline \multirow{2}{*}{$\begin{array}{l}\text { Tiempo } \\
\text { en DK }\end{array}$} & \multirow{2}{*}{$\begin{array}{l}\text { Pacientes } \\
\text { en DK (n) }\end{array}$} & \multicolumn{4}{|c|}{ Reducción crisis } & \multirow{2}{*}{$\begin{array}{c}\text { Pacientes } \\
\text { responden } \\
(\%)\end{array}$} \\
\hline & & Sin crisis & $90-99 \%$ & $50-90 \%$ & $<\mathbf{5 0} \%$ & \\
\hline 6 meses & $16 / 21$ & 2 & 8 & 3 & 3 & 62 \\
\hline 12 meses & $15 / 21$ & 5 & 7 & 1 & 2 & 62 \\
\hline 18 meses & $14 / 21$ & 5 & 7 & 2 & & 67 \\
\hline
\end{tabular}

$\mathrm{p}<0,05)$ y $64 \%$ de los pacientes estaban hipercolesterolémicos, $9 \%$ en riesgo y un $27 \%$ tenía el CT dentro de rangos normales. Al año de dieta los valores no variaron en forma significativa, pero a los 18 meses, el CT promedio disminuyó a valores de riesgo $(180 \pm 31,6 \mathrm{mg} / \mathrm{dl})$ y el porcentaje de hipercolesterolemia se redujo a $15 \%$ (tabla 2). Uno de nuestros pacientes tenía hipertrigliceridemia familiar y requirió tratamiento medicamentoso con omega-3.

Dos pacientes presentaron litiasis renal (9\%), de los cuales uno requirió cirugía para la remoción del cálculo y el otro se trató médicamente con alcalinización de la orina. En dos pacientes se pesquisó hipercalciuria.

\section{Discusión}

La dieta cetogénica ha sido usada por más de 80 años para el tratamiento de la epilepsia refractaria, demostrándose en varios estudios que, aproximadamente la mitad de los pacientes, disminuirá a un $50 \%$ el número de convulsiones, y cerca de un tercio del total disminuirá un $90 \%$ su número de crisis ${ }^{3}$. Nuestro estudio, primera experiencia local publicada, mostró mejores resultados a los descritos. Los 21 niños que entraron a este programa eran considerados "intratables". Al año de estar en dieta, 24\% estaba sin convulsiones, lo cual es mayor a lo encontrado en la literatura $(10-19 \%)^{13,14,19}$. Sólo una publicación muestra un resultado similar al nuestro ${ }^{5}$ Es muy poco probable que estos resultados sean atribuibles a remisión espontánea de la enfermedad, ya que sólo hay un estudio al respecto que encontró una tasa de remisión espontánea menor a $4 \%$ al año, cifra muy inferior a la encontrada en nuestros pacientes ${ }^{20}$.
Tabla 2. Evolución colesterol plasmático

\begin{tabular}{lcccc}
\hline & Ingreso & $\begin{array}{c}\mathbf{6} \\
\text { meses }\end{array}$ & $\begin{array}{c}\mathbf{1 2} \\
\text { meses }\end{array}$ & $\begin{array}{c}\mathbf{1 8} \\
\text { meses }\end{array}$ \\
\hline $\begin{array}{l}\text { Colesterol } \\
\text { total }(\mathrm{mg} / \mathrm{dl})\end{array}$ & 165 & 216 & 195 & 180 \\
\hline \begin{tabular}{l} 
Prueba t. *ingreso vs $6 \mathrm{~m} \mathrm{p}<0,05 * * 6 \mathrm{~m}$ vs $18 \mathrm{~m} \mathrm{p}<0,05$ \\
\hline
\end{tabular}
\end{tabular}

Hubo una buena adherencia a la dieta, comparada con otras series que publican que al año menos de la mitad de los pacientes siguen en ella ${ }^{3,14}$.

La mayoría de los efectos colaterales que produce la DK en su inicio son leves y transitorios e incluyen náuseas, vómitos, diarrea y falta de apetito, pero, en general, no afectan la aceptación del tratamiento ${ }^{14}$. En nuestra serie, la dieta fue bien tolerada, salvo en 1 paciente que debió suspenderla por diarrea persistente. Posteriormente, no hubo otras suspensiones de la dieta por intolerancia.

Respecto a la evolución del estado nutricional, llama la atención el compromiso de la talla en el seguimiento a largo plazo (18 meses). En la literatura se ha descrito que el crecimiento lineal en los pacientes con DK puede ocurrir a una velocidad menor, pero normal ${ }^{15}$. Sin embargo, otros trabajos describen una disminución significativa en la relación talla/edad después de 6 meses en dieta ${ }^{16,17}$. En nuestra serie se observó un deterioro significativo de la talla, a pesar que no hubo compromiso ponderal y que los pacientes tenían indicado aporte adecuado de proteínas, minerales y vitaminas. El mayor compromiso de la talla en nuestros pacientes podría estar dado por un mayor grado de cetosis como se ha descrito en otro estudio ${ }^{16}$, pero 
nosotros no hicimos medición cuantitativa de cetonemia, por lo que no podemos hacer la correlación.

En cuanto complicaciones, nuestro grupo no presentó más que las descritas en la literatura. La nefrolitiasis se ha encontrado en un 3-10\% de los pacientes con $\mathrm{DK}^{5,7,8,12}$, lo que coincide con lo observado en nuestros niños $(9 \%)$ y se ha asociado a la acidosis crónica, deshidratación y malabsorción de grasas y podría ser más frecuente en los pacientes que reciben inhibidores de la anhidrasa carbónica como el topiramato $^{3,12}$, pero en nuestro estudio ninguno de los 2 pacientes con cálculos había recibido este medicamento.

Se ha descrito hipercolesterolemia en 29$65 \%$ de los pacientes en $\mathrm{DK}^{7,18}$. Nuestros pacientes aumentaron los niveles de colesterol en forma significativa en los primeros meses de la dieta, pero esto fue fácilmente controlable, disminuyendo la proporción de grasas saturadas de la dieta, llegando a los 18 meses de seguimiento, a tener menor frecuencia de hipercolesterolemia que la publicada en la literatura.

En resumen, este estudio muestra una muy buena eficacia de la DK, como tratamiento de la epilepsia refractaria, y una baja frecuencia de complicaciones, por lo que debería ser considerada como alternativa terapéutica en estos pacientes.

\section{Referencias}

1.- Swink T, Vining E, Freeman J: The ketogenic diet. Adv Pediatr 1997; 44: 297-329.

2.- Wheless J: The ketogenic diet: fa(c)t or fiction. J Child Neurol 1995; 10: 419-23.

3.- Freeman J, Veggiotti P, Lanzi G, Tagliabue A, Perucca E: The ketogenic diet: from molecular mechanisms to clinical effects. Epilepsy Research 2006; 68: 145-80.

4.- Wheless J: The ketogenic diet: an effective medical therapy with side effects. J Child Neurol 2001; 16: 633-5.

5.- Kang H, Chung D, Kim D, Kim H: Early- and late- onset complications of the ketogenic diet for intractable epilepsy. Epilepsia 2004; 45: 1116-23.

6.- Ballaban-Gil K, Callahan C, O'Dell C, Pappo M, Mosh'e S, Shinnar S: Complications of the ketogenic diet. Epilepsia 1998; 39: 744-8.

7.- Ríos V, Panico L, Demartini M, Carniello M: Complicaciones en el tratamiento de la epilepsia con dieta cetogénica. Rev Neurol 2001; 33: 909-15.

8.- Kielb S, Koo H, Bloom D, Faerberg G: Nephrolithiasis associated with the ketogenic diet. The journal of Urology. 2000; 164: 464-6.

9.- Best T, Franz D, Gilbert D, Nelson D, Epstein M: Cardiac complications in pediatric patients on the ketogenic diet. Neurology 2000; 54: 2328-30.

10.- Bergqvist A, Chee C, Lutchka L, RychikJ, Stallings V: Selenium deficiency associated with cardiomyopathy: A complication of the ketogenic Diet. Epilepsia 2003; 44: 618-20.

11.- Berry-Kravis E, Booth G, Taylor A, Valentino L: Bruising and the Ketogenic Diet: Evidence fordietinduced changes in platelet function. Ann Neurol 2001; 49: 98-103.

12.- Furth S, Casey J, Pyzik P, et al: Risk factors for urolithiasis in children on the ketogenic diet. Pediatr Nephrol 2000; 15: 126-8.

13.- Vining E, Freeman J, Bellaban-Gil K, et al: A multicenter study of the eficacy of the ketogenic diet. Arch Neurol 1998; 55: 1433-7.

14.- Hemingway C, Freeman J, Pillas D, Pyzik P: The ketogenic diet: a 3- to 6- year follow up of 150 children enrolled prospectively. Pediatrics 2001; 108: 898-905.

15.- Vining E, Casey J, McGrogan J, Pyzik P, SChwert P, Freeman J: Children grow on the ketogenic diet. Epilepsia 1998; 39: 168.

16.- Peterson S, Tangney C, Pimentel-Zablah E, Hjelmgren B, Booth $G$, Berry-kravis E: Changes in growth and seizure reduction in children on the ketogenic diet as a treatment for intractable epilepsy. J Am Diet Assoc 2005; 105: 718-25.

17.- Vining E, Pyzik P, Mc Grogan J, et al: Growth of children on the ketogenic diet. Dev Med Child Neurol 2002; 44: 796-802.

18.- Delgado MR, Mills J, Sparagana S: Hipercolesterolemia associated with the ketogenic diet. Epilepsia 1996; 37: 108.

19.- Nordli D, Kroda M, Carroll J, Koenigsberger D, Hirsch L, Bruner $H$, et al: Experience with the ketogenic diet in infants. Pediatrics 2001; 108: 12933.

20.- Huttenlocher PR, Hapke RJ: A follow-up study of intractable seizures in childhood. Ann Neurol 1990; 28: 699-705. 